\title{
What happened to enterovirus D68 infections in 2015?
}

\author{
Harris $D^{1}$, Desai $\mathrm{S}^{2}$, Smieja $\mathrm{M}^{3}$, Rutherford $\mathrm{C}^{4}$, Mertz $\mathrm{D}^{1}$, Pernica JM²*
}

\begin{abstract}
Background: Enterovirus-D68 (EV-D68) was observed in association with severe respiratory disease in children in North America and around the world in the fall of 2014.

Objective: To compare fall 2014 detection rates with fall 2015 detection rates of EV-D68 in nasopharyngeal swab (NPS) samples collected for routine clinical care from a large regional laboratory in south-central Ontario.
\end{abstract}

Method: Consecutive NPS samples submitted from inpatients and outpatients in Hamilton, Niagara Region and Burlington to the Regional Virology Laboratory were tested with multiplex polymerase chain reaction (PCR) for rhinovirus/enterovirus (as a single target) and for other common respiratory viruses. All NPS samples positive for rhinovirus/enterovirus were reflexed to a lab-developed single target PCR for EV-D68 detection.

Results: In 2014, between August 1 and October 31, 566 of 1,497 (38\%, 95\%Cl 35-40\%) NPS samples were rhino/enterovirus positive, of which $177(31 \%, 95 \% \mathrm{Cl} 27-35 \%)$ were confirmed as EV-D68. In 2015, between August 1 and October 31, 472 of 1,630 (29\%, 95\% Cl 27-31\%) NPS samples were rhino/enterovirus positive, of which none $(0 \%$, upper limit $97.5 \% \mathrm{Cl} 0.8 \%)$ were confirmed to be EV-D68.

Conclusion: Based on testing results, there appears to be much less circulating EV-D68 in south central Ontario in 2015 than in 2014. Further studies would be helpful to determine if detection rates have also dramatically decreased in other regions in Canada and internationally.

\section{Affiliations}

'Department of Medicine, McMaster University, Hamilton, ON

${ }^{2}$ Department of Pediatrics, McMaster University, Hamilton, ON

${ }^{3}$ Department of Pathology and Molecular Medicine, McMaster University, Hamilton, ON

${ }^{4}$ Hamilton Health Sciences, Hamilton, ON

\section{*Correspondence}

pernica@mcmaster.ca

Suggested citation: Harris D, Desai S, Smieja M, Rutherford C, Mertz D, Pernica JM. What happened to enterovirus D68 infections in 2015?. Can Comm Dis Rep 2016;42:9-11. https://doi.org/10.14745/ccdr.v42i01a02

\section{Introduction}

Enterovirus (EV) infections are ubiquitous worldwide and are responsible for significant morbidity and mortality in both children and adults $(1,2,3)$. Though the majority of EV infections in healthy individuals are asymptomatic or associated with mild symptoms, the pathogenic potential of EV infections should not be underestimated. In East Asia, EV-71 regularly causes epidemics of hand-foot-and-mouth disease, severe neurologic disease or both (1) and intensive research has gone into the development of a vaccine against this pathogen $(4,5)$.

In August 2014, a previously rare EV serotype, EV-D68, was isolated from children hospitalized with severe respiratory disease in the American Midwest (6) and was followed by reports of EV-D68 infections across North America $(6,7,8,9,10)$ and Europe $(11,12)$. The timing of the European EV-D68 spread was similar to North America. And the A and B clades circulating in Europe were very closely related to those causing the outbreak in the United States (US) and Canada (11). Community- and hospital-based surveillance in British Columbia (BC), Alberta and Quebec documented a significant eight-fold increase in circulating EVD68 in late 2014 compared to the previous year (7). There was a total of 211 cases of EV-D68 detected in BC from August 28 to December 31, with the vast majority occurring in October and November. Estimates of the overall hospitalization rate attributable to EV-D68 were as high as 21 per 100,000 in the under-five year age group (7).

Children admitted to hospital in Canada with EV-D68 infection were shown to be predominantly male and often critically ill, with 6.8 to $23 \%$ requiring intensive care $(13,14)$. The emergence of this pathogen was felt to be a concern not only because of its association with respiratory disease but also because of the hypothesis that EV-D68 infection could lead to pediatric acute flaccid paralysis/myelitis, given the sudden temporally associated appearance across North America of numerous cases of acute flaccid paralysis or myelitis $(15,16,17)$. It has been recently re-emphasized that it is very important to promptly detect and describe outbreaks, quantify their impact, and have national surveillance of enteroviruses in Canada (18). 
Many reference laboratories in Canada now routinely use highly sensitive multiplex polymerase chain reaction (PCR)-based methods for the detection of respiratory viruses, although some reserve such tests for severe illness or surveillance. The Regional Virology Laboratory (RVL) of the Hamilton Regional Laboratory Medicine Program performs routine multiplex PCR for ten respiratory viruses, regardless of disease severity, and includes a rhinovirus/enterovirus target. The RVL tests clinical samples from acute care hospitals and urgent care centres in Hamilton, Niagara Region and Burlington, a catchment area of approximately 1.3 million inhabitants.

The objective of this study was to document the results of the RVL's nasopharyngeal specimen (NPS) testing in the fall of 2015 and compare it with results from the fall of 2014.

\section{Methods}

The RVL's respiratory panel contains a PCR target highly conserved among rhinoviruses and enteroviruses. This test is very sensitive for the detection of rhinovirus/enterovirus but definite pathogen identification requires a second test specific to enteroviruses. It was discovered in 2014 that the enterovirus-specific assay previously used at the RVL lacked the sensitivity of the rhinovirus/enterovirus assay and did not identify EVD68 reliably. A highly sensitive, laboratory-developed realtime PCR assay for a unique sequence within the VP1 gene of EV-D68 was developed.

Flocked NPS samples collected as part of routine care and transported in viral transport medium were extracted using the easyMag ${ }^{\circledR}$ platform (bioMérieux ${ }^{\circledR}$, Marcy L'Etoile, France), and tested by two multiplex PCRs (one targeting influenza $A$ and $B$, respiratory syncytial virus and rhinovirus/enterovirus and the other targeting parainfluenza types 1-3, metapneumovirus and adenovirus). All NPS testing results for the Region are entered weekly into a database containing date, age and clinical centre. Specific testing for EV-D68 was done with all NPS which tested positive for rhinovirus/enterovirus, received between August 1 and October 31 in both 2014 and 2015. Comparison between the proportion of all NPS samples positive for rhino/ enterovirus in 2014 and 2015 was done using STATA version 11.2 (College Station, Texas).

\section{Results}

From August 1 to October 31, 2014, 38\% of NPS samples $(566 / 1,497)$ were rhinovirus/enterovirus positive, of which $31 \%(177 / 566)$ were confirmed to be EV-D68. The first EV-D68 positives were found in week 32 (August 1-9). The peak occurred in week 38 (September 15-21) and the last cases were detected in week 43 (October 20-26), with no cases detected in weeks 44 and 45 (Figure 1) (13). In 2015, again from August 1 to October $31,29 \%$ of NPS samples $(472 / 1,630)$ were rhinovirus/enterovirus positive, of which zero were confirmed to be EV-D68. The overall proportion of rhinovirus/enterovirus positives in 2015 was $9 \%$ less than in $2014(95 \% \mathrm{Cl} 5.612 \%$ less, $\mathrm{p}<0.001)$. The timing of the peak of rhino/enterovirus activity in 2015 was in weeks 39 and 40, compared with week 38 in 2014. This may be due to the fact that schools started one week later in 2015 (Figure 1).

Figure 1: Weekly incidence of nasopharyngeal swabs positive for rhinovirus/enterovirus, Hamilton Regional Virology Laboratory in 2014 and 2015

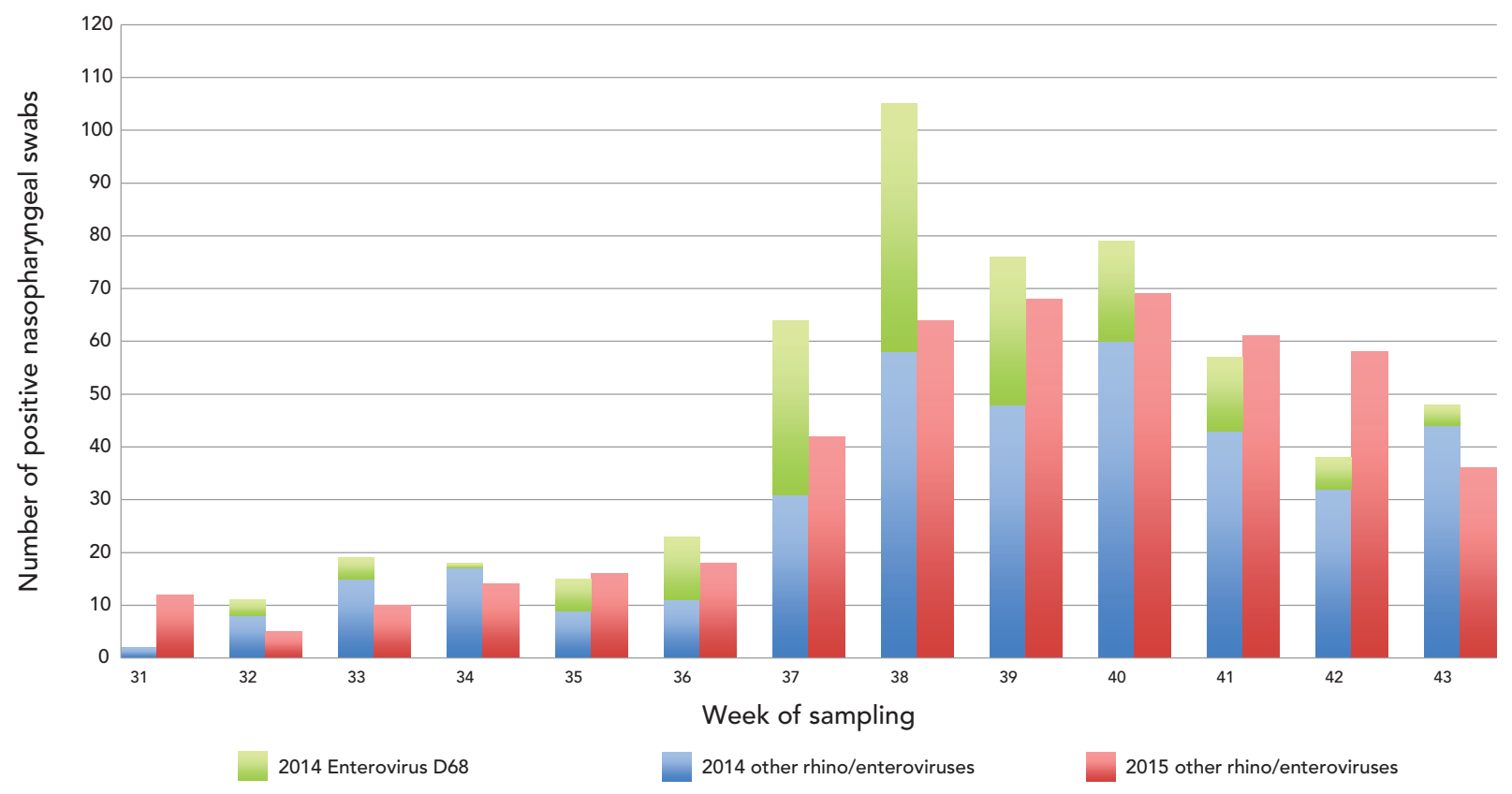

Page $10 \quad$ CCDR • January 7, 2016 • Volume 42-1 


\section{Discussion}

There has been no evidence of any significant or sustained EV-D68 transmission in the region in 2015, and the peak of rhinovirus/enterovirus season has passed. The timing of the EV-D68 peak in 2014 was consistent with typical rhinovirus/enterovirus circulation in the region; Canadian surveillance data have shown that hospitalizations for asthma exacerbations, primarily triggered by viral infections, peak 16.7 days $(95 \% \mathrm{Cl} 15.8-17.5$ days) after the first day of public school (19). There may have been much less circulating EV-D68 in 2015 simply because there were many fewer susceptible individuals than there were in 2014; alternatively, EV-D68 might have been associated with less severe disease in 2015, which would also lead to diminished recognition because of fewer presentations to medical professionals. Since the population served by the Regional Virology Laboratory is large, reflected in part by the fact that in 2014 there were almost as many EV-D68 positives in its catchment as in the entire province of $\mathrm{BC}$, it may be that other Canadian regions will also experience diminished EV-D68 activity in 2015.

There have been no cases of acute flaccid paralysis or myelitis diagnosed in our region during the current enterovirus season and the lack of circulating EV-D68 leads the authors to be cautiously optimistic that an increased incidence of neurologic disease will not be observed this year. However, the possibility of a late spike in circulating rhino/enteroviruses cannot be ruled out and therefore regional surveillance will continue. EV-D68, for now, continues to be a public health issue of relevance, given its known ability to cause respiratory disease and possible association with severe neurologic sequelae in children.

\section{Conflict of interest}

None.

\section{Funding}

None.

\section{References}

1. Ooi MH, Wong SC, Lewthwaite $\mathrm{P}$, et al. Clinical features, diagnosis, and management of enterovirus 71. Lancet Neurol. 2010;9:1097-1105.

2. Mandell GL, Bennet JE, Dolin R, eds. Principles and practice of infectious diseases. Philadelphia PA: Churchill Livingstone Elsevier; 2010.

3. Long SS, Pickering L, Prober CG. Principles and practice of pediatric infectious diseases. Philadelphia PA: Elsevier; 2008.

4. Li R, Liu L, Mo Z, et al. An inactivated enterovirus 71 vaccine in healthy children. N Engl J Med. 2014;370:829-837.

5. Zhu F, Xu W, Xia J, et al. Efficacy, safety, and immunogenicity of an enterovirus 71 vaccine in China. $\mathrm{N}$ Engl $\mathrm{J}$ Med. 2014;370:818-828.
6. Midgley CM, Jackson MA, Selvarangan R, et al. Severe respiratory illness associated with enterovirus D68 Missouri and Illinois, 2014. MMWR Morb Mortal Wkly Rep. 2014;63:798-799.

7. Skowronski DM, Chambers C, Sabaiduc S, et al. Systematic community- and hospital-based surveillance for enterovirus-D68 in three Canadian provinces, August to December 2014. Euro Surveill. 2015;20:1-14.

8. Rao S, Holzberg J, Rick A-M, et al. Enterovirus D68 in critically ill children: a comparison with pandemic H1N1 influenza. Infectious Disease Society of America Annual Conference, San Diego, CA., 2015.

9. Nolan SM, Welter J, Caylan E, et al. Enterovirus-D68 causes more severe respiratory disease than human rhinoviruses in children. Infectious Disease Society of America Annual Conference, San Diego, CA., 2015.

10. US Centers for Disease Control. Enterovirus D68. Atlanta GA: CDC; March 2015. http://www.cdc.gov/non-polioenterovirus/about/ev-d68.html.

11. Poelman R, Schuffenecker I, Van Leer-Buter C, et al. European surveillance for enterovirus D68 during the emerging North-American outbreak in 2014. J Clin Virol. 2015;71:1-9.

12. European Centre for Disease Prevention and Control. Enterovirus D68 detected in the USA, Canada, and Europe. Second update 25 November 2014. Stockholm ECDC; 2014. http://ecdc.europa.eu/en/publications/Publications/ Enterovirus-68-detected-in-the-USA-Canada-Europesecond-update-25-November-2014.pdf.

13. Mertz D, Alawfi A, Pernica JM, et al. Clinical severity of pediatric respiratory illness with enterovirus D68 as compared with rhinovirus or other enterovirus genotypes. CMAJ. 2015; In press.

14. Edwin JJ, Reyes-Domingo F, Booth TF, et al. Surveillance summary of hospitalized pediatric enterovirus D68 cases in Canada, September 2014. Can Commun Dis Rep. 2015;41:2-8.

15. Division of Viral Diseases NCFL, Respiratory Diseases CDC, Division of Vector-Borne Diseases DoH-CP, et al. Notes from the field: acute flaccid myelitis among persons aged $</=21$ years - United States, August 1-November 13, 2014. MMWR Morb Mortal Wkly Rep. 2015;63:1243-1244.

16. Messacar K, Schreiner TL, Maloney JA, et al. A cluster of acute flaccid paralysis and cranial nerve dysfunction temporally associated with an outbreak of enterovirus D68 in children in Colorado, USA. Lancet. 2015;385:1662-1671.

17. Greninger AL, Naccache SN, Messacar K, et al. A novel outbreak enterovirus D68 strain associated with acute flaccid myelitis cases in the USA (2012-14): a retrospective cohort study. Lancet Infect Dis. 2015;15:671-682.

18. Booth TF, Grudeski E, McDermid A. National surveillance for non-polio enteroviruses in Canada: why is it important? Can Commun Dis Rep. 2015;41:11-17.

19. Johnston NW, Johnston SL, Norman GR, et al. The September epidemic of asthma hospitalization: school children as disease vectors. J Allergy Clin Immunol. 2006; 117:557-562. 